EPJ Web of Conferences 32, 04013 (2012)

DOI: $10.1051 /$ epjconf/20123204013

(C) Owned by the authors, published by EDP Sciences, 2012

\title{
Measurements on Fast switches and combiners (FADIS-BC) for High-Power Millimeter-wave beams based on dielectric beam splitters
}

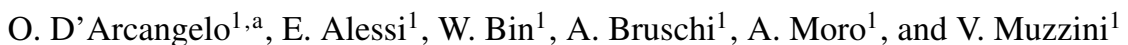

Istituto di Fisica del Plasma "Piero Caldirola”, CNR EURATOM-ENEA Association via R. Cozzi 53, 20125 Milano, Italy

\begin{abstract}
An upgraded version of a quasi-optical diplexer combiner, based on a resonating system coupling two transmission lines using three Dielectric Beam Splitters, has been realized and tested. This device is principally thought to combine power coming from different transmission lines into a single output or to switch the injected power between different outputs, but it could also be used as mode filter or in line viewing system for Electron Cyclotron Emission (ECE) or Collective Thomson Scattering (CTS) diagnostics. The design was implemented in order to link two transmission lines of the ECRH system on FTU, for power combination of two beams into a single line of the new ECRH launcher recently installed. This device is based on Dielectric Beam Splitters (DBS); it has been tested at low power in order to confirm the splitting ratio foreseen from simulation and its efficiency has been evaluated measuring the beam exiting the system. The design and the characterization of the diplexer combiner are reported in this paper.
\end{abstract}

\section{Introduction}

The increasing number of transmission lines and the raising EC installed power foreseen in the next generation fusion machines stimulates the development of switching-combining devices. They are able to combine power from different sources into one single output channel and to redirect it choosing between multiple transmission lines [1,2], with a switching velocity of a few ms (mechanical path tuning) or even less (frequency tuning of the sources). Initially these devices were thought to enhance the efficiency during Neoclassical Tearing Modes (NTM) control experiments and to make the ECRH transmission systems extremely flexible in view of a more efficient EC Heating and Current Drive. Many different designs have been proposed, realized and tested during past years [3,4]: they could be divided mainly in Mach-Zehnder interferometers and quasi optical four-ports resonators (figure 1)
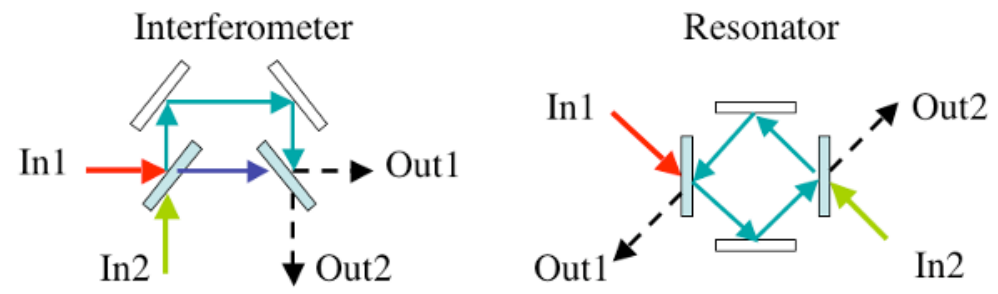

Fig. 1. General scheme of the resonating and interferometric configuration of the four-port device.

\footnotetext{
a e-mail: darcangelo@ifp.cnr.it
} 
realized with coupling gratings, dielectric beam splitters (DBSs) or square waveguide splitters. Later it was realized that they could also be used for in-line ECE and CTS viewing system and moreover can act as mode filters, opening the possibility to realize tools for qualifying the TL/RF source beam mode purity and to evaluate the total power in the HE11 waveguide mode.

In recent years a diplexer design has been extensively studied and characterized at IFP-CNR [5]. This design is mainly based on a resonating system and made up of two or three DBSs and suitable optics (figure 2). Each DBS is made of a water-free silica layer, three quarter of wavelength thick at 140 $\mathrm{GHz}(\mathrm{n}=1.95, \mathrm{~d} \simeq 0.88 \mathrm{~mm}, \tan \phi=5 * 10-4)$. The device consists of also 4 focusing mirror whose center is placed in a plane perpendicular to the direction of propagation of the entering beam and two plane mirror positioned in front of the two DBS working as input/outputs (figure 3, left). A first prototype [5, 6] was developed to couple two transmission lines on the old FTU antennas, while after the installation of the new launcher in FTU $[7,8]$, the design has been revised to demonstrate the applicability of the concept to the new requirements: the increased distance between the waveguide transmission lines and the different output polarization angle required to inject from the new antenna a linear O-mode polarization in the plasma. This work reports the design of the device (section 2), then the evaluation of the performance in terms of splitting ratio by means of frequency tests (section 3) finally describing the results of the beam pattern measurements (section 4), performed in order to evaluate the efficiency of the prototype.

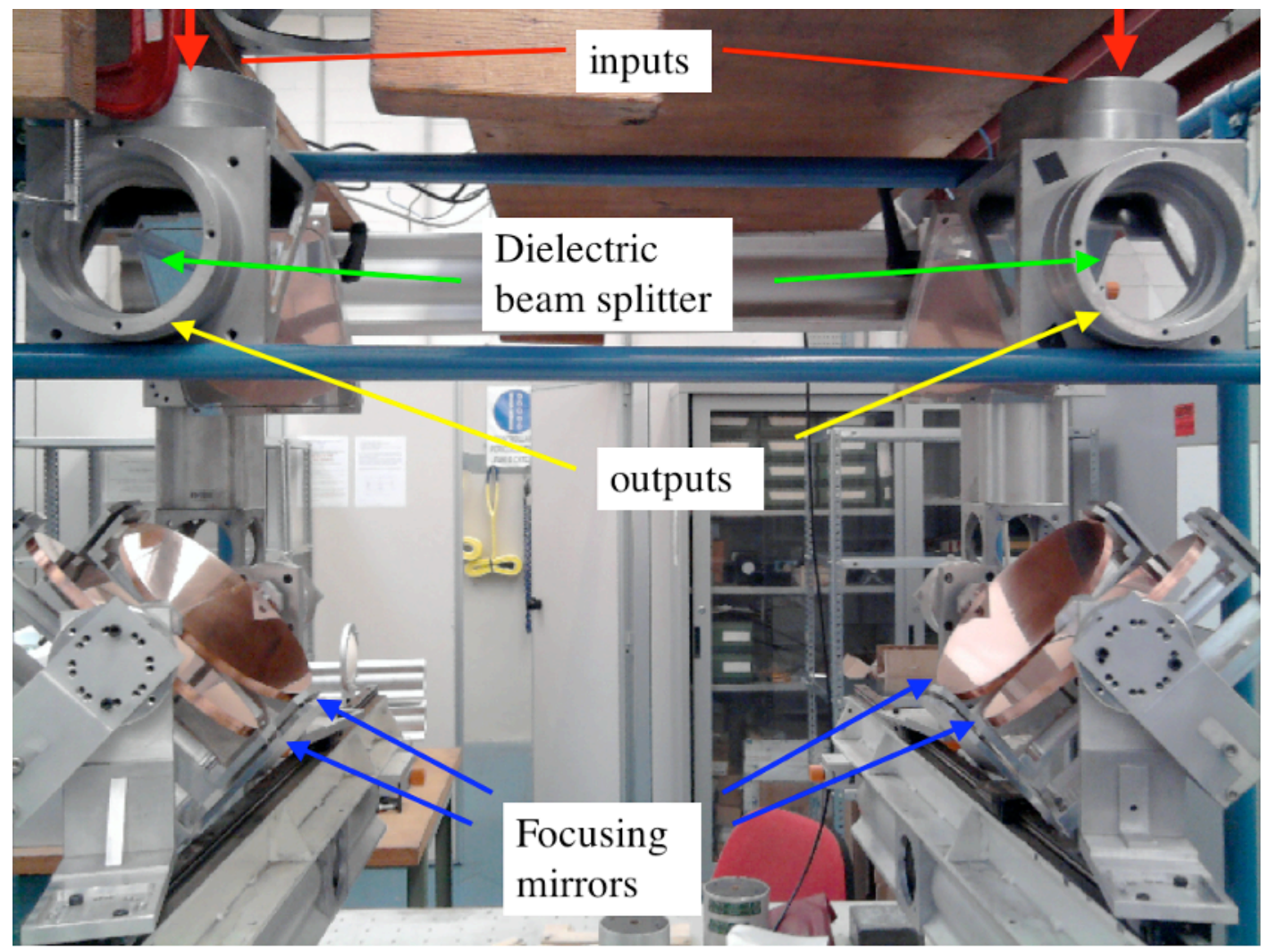

Fig. 2. Picture of the prototype combiner realized at IFP-CNR.

\section{Conceptual design}

One of the most important characteristics of DBSs is the dependence of the transmission coefficients on the input polarization for non-perpendicular incidence; therefore splitting ratios between different input/output ports change with the input polarization in devices working with DBSs. A theoretical 
model was developed in order to predict the performances of the different configurations with different input polarization angles [6] and this model was extensively checked with the previous version of diplexer combiner realized at IFP-CNR. The theoretical model confirmed that the present design can be modified to obtain good splitting ratios for the required output polarizations. In fact, it has been found that, for a fixed geometry of the diplexer, good splitting ratios can be achieved by tilting the polarization of the input beams until the polarization of the circulating beam is incident to the central DBS parallel to its surface (figure 3). Then, for fixed polarizations, by changing the geometry, and in particular the ratio $\mathrm{L} / \mathrm{D}$, the design can be adapted to a fixed linear polarization required at the end of ECH transmission lines. For convenience, as in the previous version of prototype, the two beam splitters placed in front of the inputs are mounted on mock-ups of the miter bends of the FTU ECRH lines, as a replacement of the mitre-bend mirrors. This is the way in which the insertion on the transmission line could be more convenient, requiring only a substitution of DBS with the original mitre-bend mirrors to return to the standard waveguide configuration. A diplexer, designed for a given input polarization angle in IN1(figure 3), has the same performaces for a second beam injected in IN2, due to the symmetries of the system, provided that the beam entering from IN2 has the symmetric polarization with respect to the beam entering in IN1. In figure 3 (right) the front view of the polarization resulting at the two outputs and for which characteristics have been measured. The present design, when used for the combination of two beams into a single line of the new launcher, does not require additional mirrors to adjust the polarization required in the second output. Additional mirrors could be added to provide the required polarization simultaneously in both outputs in experiments where the $\mathrm{ECH}$ power is switched between two lines directed to different launchers, as for experiments requiring fast switching between different ECH applications.

In principle, a given diplexer would work only if the input and output polarizations are constant. However, it has been found that a small variation of the input polarization results in a similar change in the output one, weakly affecting the splitting ratio characteristics. In Figure 3 the maximum and minimum transmission efficiencies at both outputs, calculated by the theoretical model are plotted by varying the input polarization angle in IN1. Calculations show good splitting ratio also for input/output polarization angles different (within $\pm 8^{\circ}$ ) from the design reference $\left(15.4^{\circ}\right.$ ). The robustness of the combiner characteristics to variations in input polarization angle is shown in (figure 4). In the version presented in this work, besides the upgraded geometry with respect to the previous version the four focusing elliptical mirrors were also replaced with larger mirrors, in order to minimize losses due to diffraction.

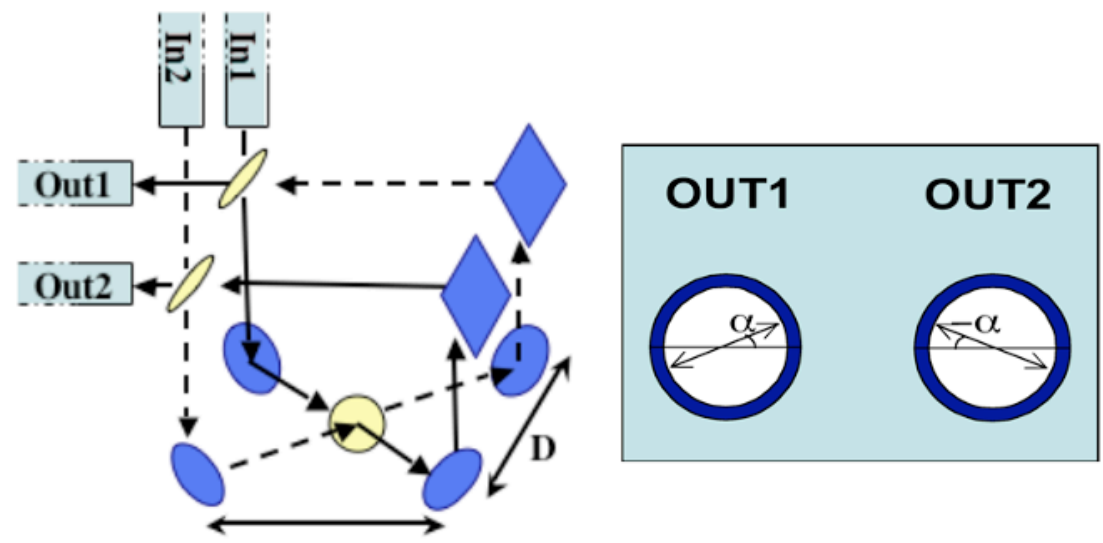

L

Fig. 3. Left: Schematic view of the quasi optical diplexer combiner ( $L=190 \mathrm{~mm}, \mathrm{D}=700 \mathrm{~mm})$; the yellow circles represent the dielectric beam splitters, the blue squares represent the plane mirrors and the blue circles are the focusing mirrors. Right: Sketch of the diplexer front view. Nominal polarizations are shown with respect to the horizontal plane in the laboratory frame. Present prototype (arrows) works with $\alpha=15.4^{\circ}$ 


\section{Frequency measurements}

The millimeter-wave gaussian beam used for low-power measurements was obtained with a hornmirror combination. Before mounting the launcher in place for measurements, the beam central position was measured at a distance of around one meter from the launcher end. Afterwards, disconnecting the source (a gunn) from the launcher, a laser was placed firmly on the same mounting of the gaussian launcher behind the horn. The light beam was passing through the small waveguide aperture in the horn and the laser was aligned to the position of the center of the gaussian beam already measured. In
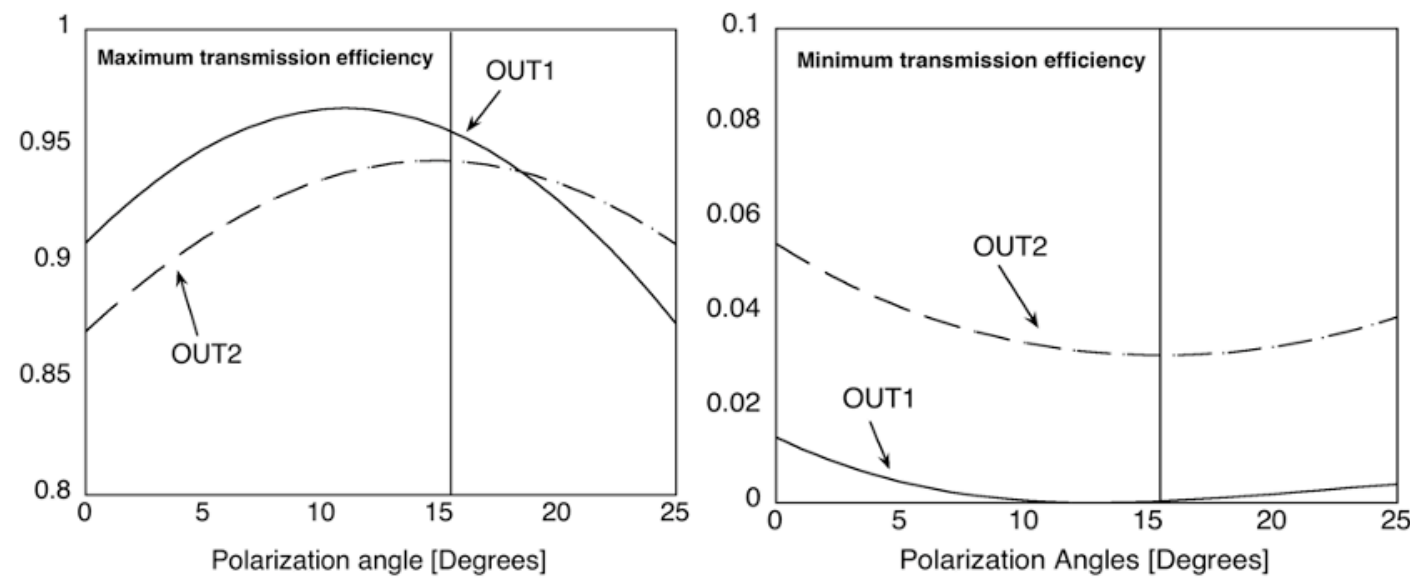

Fig. 4. Maximum (left) and minimum (right) transmission efficiencies at both outputs as function of the input polarization angle. Output polarizations vary accordingly with input polarization.

this way a good matching between the optical beam and the direction of the RF beam is obtained. After placing the gaussian launcher in its final position, the setup of the diplexer-combiner optics began with the optical alignment; it was essential to assure a high accuracy in positioning all the different components of the system (focusing and plane mirrors, DBS) aligned with the laser beam. Targets were prepared and placed on every single mirror, on the mitre bends mock-ups (at the place where the DBS would be mounted) and on the output ports. The position of each component was adjusted until, at the end of the alignment process, the laser spot was centered on each target inside the structure and at the two outputs. After this first step, the device was ready for frequency tests, with the laser still mounted and the gunn placed in position and connected to the horn.

The idea was to check the theoretical predictions concerning the splitting ratio of the system but also to check the performed optical alignment. The splitting ratio of the diplexer combiner configuration, expected to be periodical with frequency, was evaluated with frequency scans only from a qualitatively point of view. In fact these measurements were made placing the receiver in a fixed position facing the outputs, thus the signal was representative of the central power density of the beam exiting the device. All measurements were made using an AB mm Vector Network Analyzer (VNA) and the frequency sweeps were performed over some periods of the diplexer characteristic around $140 \mathrm{GHz}$. The signal is inserted in the diplexer combiner from one single input (IN1 in figure 2) and the two outputs are measured simultaneously during frequency sweeps. An additional fine tuning of the third DBS (at the centre of the resonator) was required to optimise the shape and the symmetry of the two output power curves with respect to frequency during the sweeps. In fact, as the theoretical model predicts small displacements (of about $50 \mu \mathrm{m}$ ) of the central DBS cause asymmetric frequency characteristics and a (relatively small) performance reduction.

Due to the enlarged mirror dimensions the diffraction losses were expected to be extremely small and have been at first neglected to evaluate the diplexer characteristics. Therefore measurements have been normalized with respect to the total power exiting from both outputs at the nominal linear polariza- 
tion. The normalized data obtained after an accurate alignment are shown in figure 5. The measured data clearly indicate that the device behaves as expected; at each port the output power varies of more than an order of magnitude with frequency and the splitting ratio seems very encouraging in view of application of the device in a high power ECRH system.

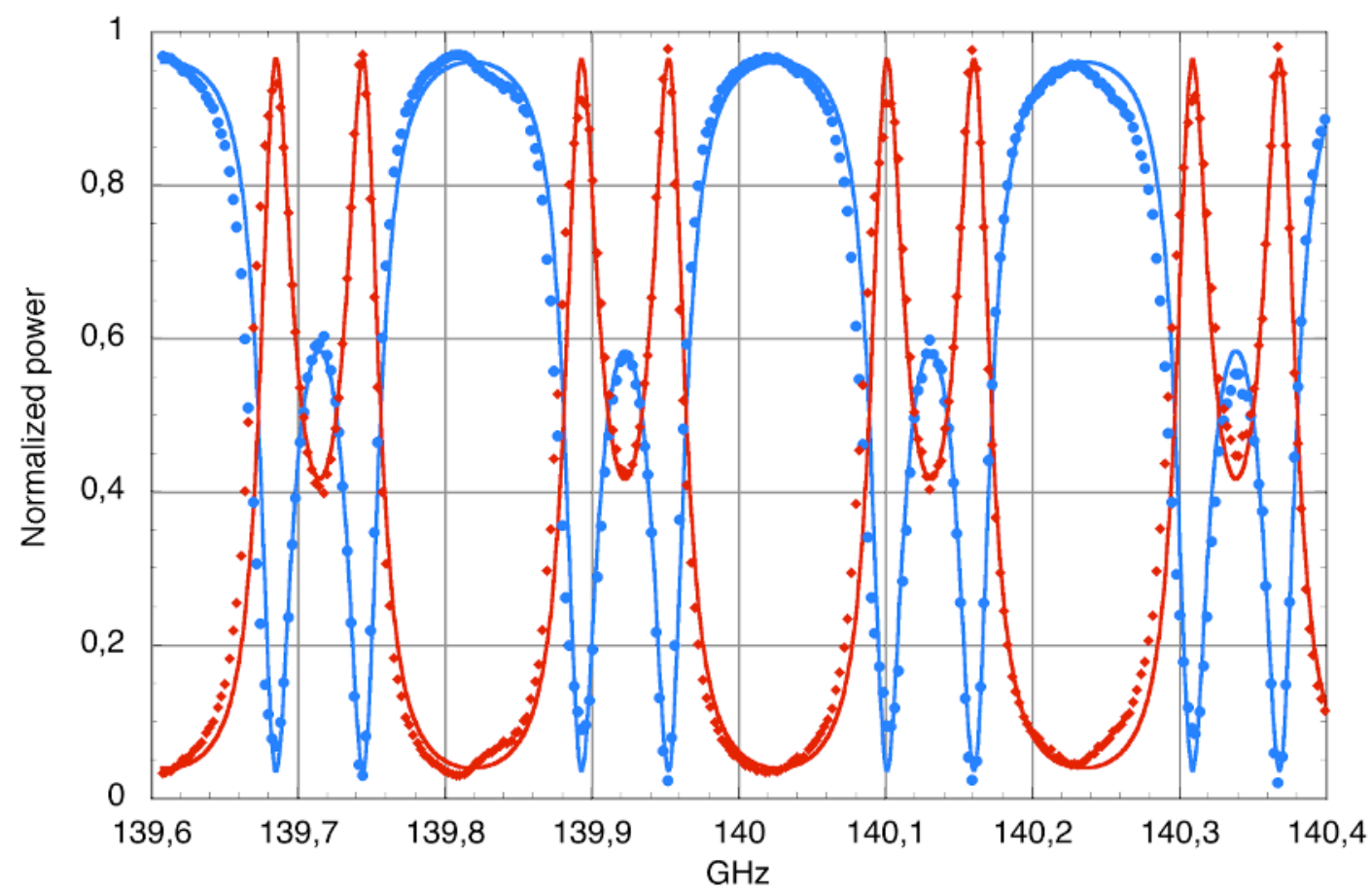

Fig. 5. Signal measured (symbol) at output 1 (blue circles) and output 2 (red squares) compared with the simulated curve (blue continuos line out1, red line out2)

\section{Power measurements}

The last step for the characterization of the diplexer-combiner was the evaluation of its efficiency in terms of power transmission; the output power was evaluated measuring the beam pattern at the two outputs at different frequencies. The beam is evaluated on a planar surface of 100x100 mm extension, perpendicular to the direction of propagation. At the edge of this surface the beam is more than $30 \mathrm{~dB}$ below its maximum, thus the power can be measured with very good accuracy integrating the power density measured on the surface. All patterns were acquired at fixed frequencies, where the maximum power is expected either at output 1 or at output 2 (figure 5). Examples of the beam measured at output 1 and 2 are shown in figure (6). In order to evaluate the losses of the device, the measured power was normalized with the input power at the same frequency. The reference beam is measured substituting the DBS in front of the gaussian launcher with a reflecting plane mirror; in this way all the power exiting the source is directed towards OUT1 and detected by the receiver. The dimensions of the measured beam were evaluated fitting the data with a gaussian function and results were compared to the one foreseen from the design. The beam radii obtained from the measured profiles are somewhat larger than expected at OUT1 (up to 13\%) and in better agreement with the design value at OUT2, as expected due to the filtering capabilities of the combiner in the resonant conditions. Evaluating the power of the beam and normalizing it as described above, the following results were obtained: the maximum power transmitted at OUT1 is around $92 \%$ with respect a predicted value of $96 \%$ while in OUT2 the maximum power transmitted is around $90 \%$ with respect to $94 \%$. Thus the experimental results show a transmission efficiency around $4 \%$ less than the expected value. Due to the long time 

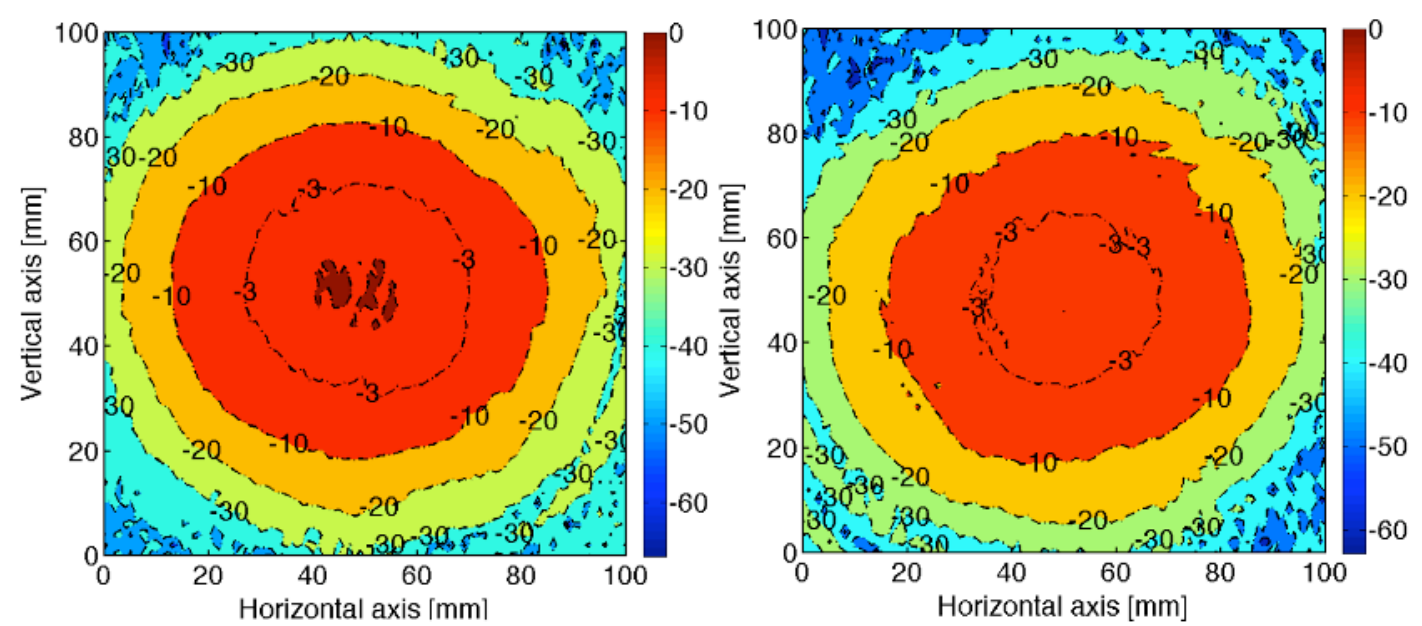

Fig. 6. Beam power measured at output 1 (left) and output 2 (right). Both beam were measured at a frequency where the signal reported in figure 5 reaches its maximum value.

required to perform the beam power measurements, unavoidable thermal drifts of the measurement chain and of the mechanical structure supporting the mirrors of the present prototype prevented a more precise and stable enough estimate of the power losses. In fact the uncertainty of the measurements $(\leq$ $5 \%$ ) is still comparable with the expected total power losses.

\section{Conclusions}

An upgraded version of a resonant diplexer-combiner based on dielectric beam splitters was realized and tested at low power. It has been designed to couple two transmission lines of the ECRH system of FTU into a single line of the new launcher recently installed on FTU. The theoretical splitting ratio (periodical with frequency) foreseen for the realized design has been confirmed by frequency scans around $140 \mathrm{GHz} \pm 0.4 \mathrm{GHz}$, covering some resonance periods. The efficiency of the system has been measured by evaluating the beam at the frequency of the maximum power at either output. The beam power has been normalized with respect to the power exiting the source; the results obtained shown that the efficiency of the system was around $92 \%$ at output 1 and $90 \%$ at output 2 , around $4 \%$ less than the foreseen theoretical values. A more stable setup allowing a better alignment accuracy is expected to improve the achievable efficiency.

\section{References}

1. W. Kasparek et al. Fusion Science and Technology, Vol. 52, (2007), pp. 281-290 .

2. A. Bruschi et al., Fusion Science and Technology Vol. 53 (2008), p.97.-103

3. W. Kasparek et al. , Fus. Eng. Des., Vol. 84, (2009) Pages: 1002-1005

4. A. Bruschi et al., IEEE Trans. Plasma Sci. Vol. 38, (2010), pp 1427-1438

5. D’Arcangelo O. et al. Fusion Engineering and Design, Vol. 86, (2011), Pages 920-924

6. Bruschi A. et al. IRMMW-THz 2010 -35th International Conference on Infrared, Millimeter and $\mathrm{THz}$ Waves, Rome (Italy), 2010 Article number 5612310

7. A.Bruschi et al., Fusion Science and Technology vol. 55, (2009), pp.94-107

8. W. Bin et al., Fusion Engineering and Design Vol. 84 (2009), pp. 451-456 